\title{
Effect of UV Irradiation and Temperature on Free Radical Properties in Dehydrocholic and Ursodeoxycholic Acids: An EPR Study
}

\author{
Małgorzata Dołowy, ${ }^{1}$ Pawel Ramos, ${ }^{2}$ and Barbara Pilawa ${ }^{2}$ \\ ${ }^{1}$ Department of Analytical Chemistry, Faculty of Pharmacy, Medical University of Silesia in Katowice, 4 Jagiellońska Street, \\ 41-200 Sosnowiec, Poland \\ ${ }^{2}$ Department of Biophysics, Faculty of Pharmacy, Medical University of Silesia in Katowice, 8 Jedności Street, \\ 41-200 Sosnowiec, Poland
}

Correspondence should be addressed to Małgorzata Dołowy; mdolowy@sum.edu.pl

Received 26 May 2014; Revised 22 August 2014; Accepted 7 September 2014; Published 25 November 2014

Academic Editor: Franca Morazzoni

Copyright (C) 2014 Małgorzata Dołowy et al. This is an open access article distributed under the Creative Commons Attribution License, which permits unrestricted use, distribution, and reproduction in any medium, provided the original work is properly cited.

The effect of UV irradiation and temperature on the formation and properties of free radicals in two pharmaceutical important bile acids, such as dehydrocholic (DH) and ursodeoxycholic acids (UDC), was examined. Electron paramagnetic resonance (EPR) spectroscopy was applied to determine the paramagnetic character of UV irradiated and thermally sterilized drugs. Thermal and UV irradiation sterilizations of both compounds were carried out at different conditions according to pharmaceutical norms. The performed EPR measurements of UV irradiated and thermally sterilized DH and UDC samples proved the existence of the complex free radical systems in examined bile acids. Significant influence of UV irradiation in comparison with applied thermal sterilization on free radical concentrations in DH and UDC samples was observed. The results pointed out that thermal method is most suitable for bile acid sterilization. Therefore, this kind of sterilization should be applied in practice.

\section{Introduction}

Electron paramagnetic resonance (EPR) spectroscopy is a known electromagnetic technique used for recording paramagnetic centers, such as free radicals [1-5]. Thus, it has application in different research fields like medicine and pharmacy, for example, to characterize the mechanism of pharmacological action of drugs or to estimate the effect of radiation on drug delivery system and also on its stability in solid state and in aqueous solutions, respectively [1,6-13]. Recently, the current literature review proves various applications of EPR spectroscopy in pharmacy, for example, to examine the stability of UV irradiated and thermally sterilized drugs, especially antibiotics [6, 13] and glucocorticosteroids [7]. Because the sterilized antibiotics, especially coming from anthracycline family, such as doxorubicin, daunorubicin, epidoxorubicin, are widely applied in anticancer therapy (e.g., in leukemia and breast cancer) [13], there is a need to examine their vulnerability to thermal and UV irradiation prior use. Similar requirements should be fulfilled in the case of steroid drugs which belong to the most important anti-inflammatory, antiallergic, and immunosuppressive agents, used in dermatology and ophthalmology in form of sterile pharmaceutical formulations, like, for example, prednisolone [7, 14].

The aim of this work was to examine the impact of temperature and UV irradiation on the formation and also properties of free radicals in two pharmaceutical important bile acids, such as dehydrocholic acid (DH) and ursodeoxycholic acid (UDC). Both compounds are members of a group of biologically active steroids as can be seen from Figures 1 and 2. They are widely used as a cholagogue and hydrocholeretic agents in pharmacotherapy of different liver and gallstone diseases. The literature on EPR spectroscopic study of ursodeoxycholic and dehydrocholic acids related to their paramagnetic properties and also estimation the stability of both compounds after exposition to UV irradiation or high 


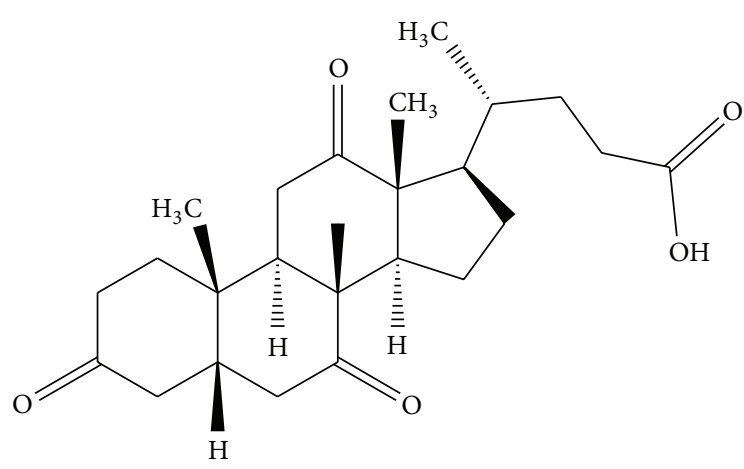

(a)

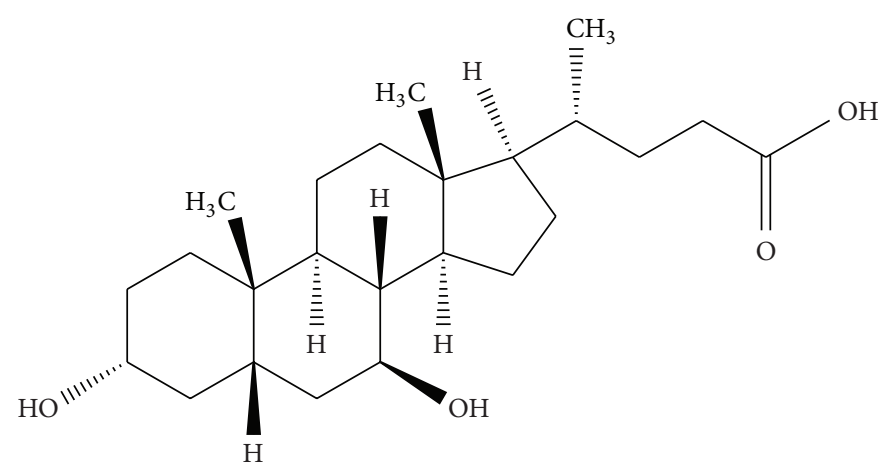

(b)

FIgURE 1: Chemical structure of dehydrocholic acid DH (a) and ursodeoxycholic acid UDC (b).

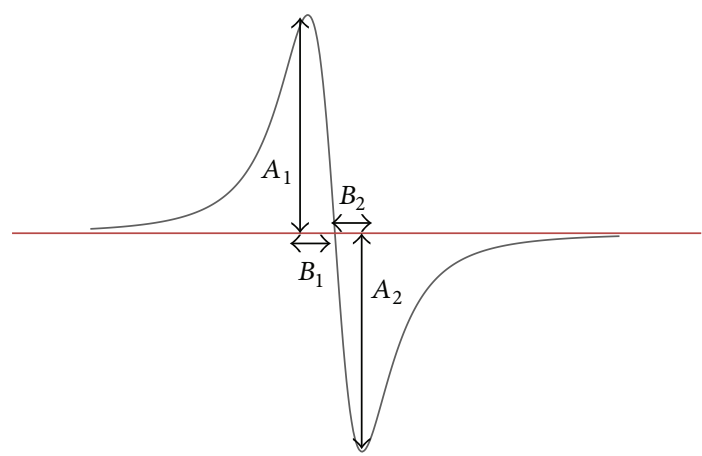

FIGURE 2: Example of EPR spectrum.

temperature is relatively sparse [15-17]. To our knowledge, until today none of the papers describing the effect of UV irradiation and also thermal sterilization on paramagnetic properties of DH and UDC in solid state has been reported. Therefore, the main purpose of this study was to assess the possibility of applying EPR spectroscopic method to describe the influence of UV irradiation and also temperature on the generation and also properties of free radicals in two pharmaceutical important bile acids: dehydrocholic and ursodeoxycholic, when they are exposed to UV light as well as to high temperature in the solid state.

The thermal and UV irradiation sterilizations were fully evaluated with regard to obligatory procedures designed for sterility assurance required in quality and quantity control of pharmaceutical preparations (according to Pharmacopoeia) [14]. The kind and also the concentration of free radicals formed in DH and UDC samples as a result of their exposure to UV irradiation and to higher temperature were determined. In order to assess the applicability of EPR spectroscopic method to determine the effect of UV irradiation as well as thermal sterilization on the generation and also the properties of free radicals in pharmaceutical preparations of tested bile acids, the results obtained for examined compounds in substantia were compared with that determined for commercial products of DH and UDC.

\section{Experimental}

2.1. Samples. DH (dehydrocholic acid, Cat. number 30830) and UDC (ursodeoxycholic acid, Cat. number. U5127) in substantia were obtained from Sigma-Aldrich (St. Louis, MO, USA). The following commercial preparations containing $\mathrm{DH}$ in form of tablets (250 mg of DH per tablet) and also UDC capsules (150 mg of UDC per capsule) were from Polish pharmaceutical market.

2.2. Sample Preparation. UV irradiated and thermally subjected samples of dehydrocholic and ursodeoxycholic acids were tested. The following times of UV irradiation process 30 minutes and 60 minutes were used, respectively. The UV irradiation was done by Medison 250 lamp with 4 radiators and with power of $20 \mathrm{~W}$. The UV irradiation with waves of $\lambda: 315-400 \mathrm{~nm}$ was performed from the distance of lamp to the sample of $30 \mathrm{~cm}$. Thermal sterilization of DH and UDC was carried out according to the Polish Pharmacopoeia Norms [14] required for drug sterilization at the following conditions: $T=160^{\circ} \mathrm{C}$ and $t=120$ minutes, $T=170^{\circ} \mathrm{C}$ and $t=60$ minutes, $T=180^{\circ} \mathrm{C}$ and $t=30$ minutes, where $T$ is temperature of this experiment and $t$ is time of heating. Heating process of powdered samples was performed in dryer supplied by Memmert firm (Schwabach, Germany) in hot air with air circulation.

2.3. EPR Measurements. EPR spectra of tested compounds were recorded for the samples placed in the thin walled glass tubes with the external diameter of $1 \mathrm{~mm}$. The EPR measurements were done for UV irradiated (during 30 minutes and 60 minutes) dehydrocholic and ursodeoxycholic acids and also for their samples which were thermally sterilized at the following temperatures and times: at $160^{\circ} \mathrm{C}$ during $120 \mathrm{~min}$ utes, at $170^{\circ} \mathrm{C}$ during 60 minutes, and at $180^{\circ} \mathrm{C}$ during 30 minutes.

EPR spectroscopic analysis of examined compounds was carried out at X band $(9.3 \mathrm{GHz})$ using EPR spectrometer produced by Radiopan Firm (Poznań, Poland). The magnetic modulation of $100 \mathrm{kHz}$ was used. Microwave frequency was 
TABle 1: Parameters of the EPR spectra of UV irradiated dehydrocholic acid (DH) and also ursodeoxycholic acid (UDC).

\begin{tabular}{llccccccc}
\hline \multirow{2}{*}{ Compound } & $\begin{array}{c}\text { Time of } \\
\text { sterilization }\end{array}$ & $\begin{array}{c}A \text { (a. u. }) \\
{[ \pm 0.02]}\end{array}$ & $\begin{array}{c}I \text { [a. u. }] \\
{[ \pm 0.02]}\end{array}$ & $\begin{array}{c}\Delta B_{\mathrm{pp}} \\
(\mathrm{mT}) \\
{[ \pm 0.02]}\end{array}$ & $\begin{array}{c}N \times 10^{17} \\
{[\mathrm{spin} / \mathrm{g}]} \\
{[ \pm 0.02]}\end{array}$ & $\begin{array}{c}A_{1} / A_{2} \\
{[ \pm 0.02]}\end{array}$ & $\begin{array}{c}B_{1} / B_{2} \\
{[ \pm 0.02]}\end{array}$ & $\begin{array}{c}g \\
{[ \pm 0.0002]}\end{array}$ \\
\hline \multirow{2}{*}{$\mathrm{DH}$} & 30 minutes & 0.34 & 60.60 & 1.54 & 169.10 & 1.04 & 0.99 \\
& 60 minutes & 0.43 & 63.80 & 1.46 & 178.10 & 1.10 & 1.52 & 1.9982 \\
\hline \multirow{2}{*}{ UDC } & 30 minutes & 0.32 & 87.30 & 2.08 & 243.80 & 0.75 & 0.67 & 1.9985 \\
& 60 minutes & 0.38 & 114.50 & 2.08 & 319.70 & 0.56 & 1.31 \\
\hline
\end{tabular}

Notes. A-amplitude of signal; $I$-integral intensity; $A_{1} / A_{2}$ and $B_{1} / B_{2}$-the asymmetry parameters; $\Delta B_{\mathrm{pp}}$-line-width of EPR signal; $N$-free radical concentrations; $g$-factor.

measured by MCM101 recorder of Eprad Firm (Poznań, Poland). The first-derivative EPR spectra were measured by means of the Rapid Scan Unit of Jagmar Firm (Kraków, Poland) which was connected with the EPR spectrometer. The numerical acquisition of the individual EPR spectra was done during the time of 1 second. The EPR spectra were recorded with microwave power of $2.2 \mathrm{~mW}$. This low microwave power was used to avoid microwave saturation effect of EPR lines. The total microwave power produced by klystron was $70 \mathrm{~mW}$, and the application of $15 \mathrm{~dB}$ attenuation makes it possible to decrease the microwave power to $2.2 \mathrm{~mW}$.

The analyses of EPR spectra were performed by use of spectroscopic program of Jagmar Firm (Kraków, Poland) and also LabVIEW 8.5 program of National Instruments Firm.

The following parameters of EPR spectra: $g$-factor, $A-$ amplitude, $I$-integral intensity, and linewidth $\left(\Delta B_{\mathrm{pp}}\right)$, were determined as was described elsewhere $[6-8,10-12]$.

$g$-factor, was calculated from the paramagnetic resonance conditions according to the formula:

$$
g=\frac{h v}{\mu_{B} B_{r}}
$$

where $h$-Planck constant, $\nu$-microwave frequency, $\mu_{B}-$ Bohr magneton, and $B_{r}$-induction of resonance magnetic field.

The integral intensities of the EPR lines of examined steroids $(I)$ and ultramarine $\left(I_{u}\right)$ were calculated by double integration of the first-derivative EPR spectra. Free radical concentrations $(N)$ in the studied samples were determined based on the proportional relationship with integral intensity of the EPR lines [6-8, 10-12]. Sample integral intensities in EPR spectra were examined and compared with an ultramarine reference. A ruby crystal $\left(\mathrm{Al}_{2} \mathrm{O}_{3}: \mathrm{Cr}^{3+}\right)$ permanently placed in the resonance cavity was used as the second reference. Amplitudes of EPR lines of the ruby crystal were detected and compared with the samples and the ultramarine control. The concentration of free radicals $(N)$ in UV irradiated and thermally subjected samples of dehydrocholic and ursodeoxycholic acids was calculated as follows:

$$
N=\frac{N_{u}\left[\left(W_{u} A_{u}\right) / I_{u}\right]}{[I /(W A m)]},
$$

where $N_{u}$-the number of the paramagnetic center in the ultramarine reference $\left(1.9 \times 10^{19}\right) ; W, W_{u}$-the receiver gains for the sample and the ultramarine; $A, A_{u}$-the amplitudes of ruby signal for the sample and the ultramarine; $I, I_{u}$-the integral intensities for the sample and ultramarine; $m$-the mass of the sample.

In order to determine the effect of microwave power on EPR spectra, the attenuation of microwaves was increased from 0 to $15 \mathrm{~dB}$. EPR spectra were measured with microwave power $(M)$ in the range of $2.2-70 \mathrm{~mW}$. Additionally, the amplitudes $(A)$ and linewidth $\left(\Delta B_{\mathrm{pp}}\right)$ of obtained spectra were determined as is shown in Figure 2.

\section{Results and Discussion}

The EPR measurements were done at room temperature for nonirradiated and irradiated samples of dehydrocholic and also ursodeoxycholic acids in solid state. Analysis of EPR spectra obtained after UV irradiation sterilization was performed by the means of spectroscopic program of Jagmar Firm (Kraków, Poland) and LabView 8.5 program of National Instruments Firm. The following parameters of these spectra: $A$-amplitude of signal, $I$-integral intensity, $A_{1} / A_{2}$ and $B_{1} / B_{2}$-the asymmetry parameters, $g$-factor, $\Delta B_{\mathrm{pp}}-$ linewidth of EPR signal, and also $N$-free radical concentrations determined in the UV irradiated samples of DH and UDC, were summarized in Table 1. EPR spectra were not detected in the original samples of studied compounds. Thus, it could be concluded that the paramagnetic effect does not exist in the nonirradiated bile acid samples. The EPR spectra were recorded in the examined samples after UV irradiation treatment. The exemplary EPR spectra for UV irradiated DH and UDC during the respective times: 30 and 60 minutes, are performed in Figure 3. As it results from Figure 3, these spectra are lightly asymmetric. Their multicomponent character and nonhomogenous properties can cause this asymmetry. The linewidth $\left(\Delta B_{\mathrm{pp}}\right)$ of EPR spectra of UV irradiated dehydrocholic and ursodeoxycholic acids are in the range of 1.46-1.54 for DH and 2.08 for UDC (Table 1). The wide EPR signals (above $1 \mathrm{mT}$ ) confirm a small distance between the formed free radicals and indicate strong dipole-dipole interactions in the studied compounds. Prolongation of the time of UV irradiation sterilization did not change the value of $\Delta B_{\mathrm{pp}}$ for UDC. In the case of $\mathrm{DH}$, the time of UV irradiation slightly affects the linewidths of EPR spectra. This fact shows that free radicals present in both samples are rather stable. Amplitudes $(A)$ of EPR lines which 


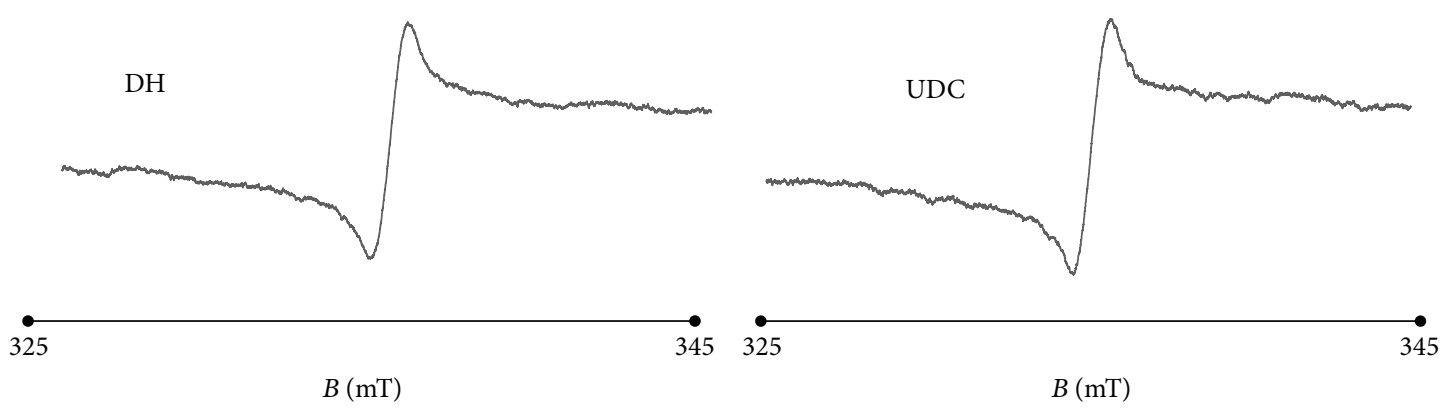

(a)

(c)

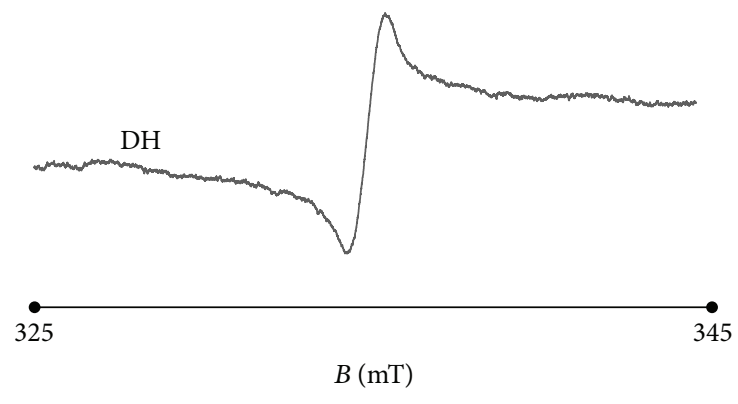

(b)

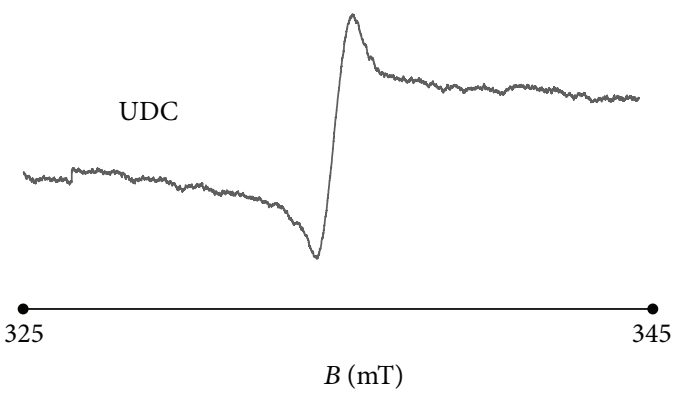

(d)

FIGURE 3: EPR spectra of DH and UDC (UV irradiated) during 30 minutes (a), (c) and also during 60 minutes (b) and (d).

are listed in Table 1 increase slowly with the increase of the time of sterilization of investigated compounds. The concentration of free radicals $(N)$ in the UV irradiated dehydrocholic acid (during 30 and 60 minutes) were about $169.10 \times$ $10^{17} \mathrm{spin} / \mathrm{g}$ and $178.10 \times 10^{17} \mathrm{spin} / \mathrm{g}$, respectively (Table 1 ). The free radical concentrations in the UV irradiated ursodeoxycholic acid changed from $243.80 \times 10^{17}$ to $319.70 \times$ $10^{17} \mathrm{spin} / \mathrm{g}$. It points that UDC is more sensitive on exposition to UV irradiation in relation to DH. We can suggest that existence of free radicals in UV irradiated drugs and bulk substances, such as bile acids will be one of the main problem which should be resolved. Our investigations indicate that optimization of UV sterilization conditions including the time of sterilization allows reducing the amount of generated free radicals in examined compounds. The data presented in Table 1 demonstrate that the optimal time for the exposition of investigated DH and also UDC to UV irradiation should be 30 minutes. In order to prove the impact of UV irradiation on free radical concentrations in bile acids, the results obtained for UV irradiated reference standards of DH and UDC were compared with those determined for commercially available pharmaceutical formulations containing individually DH and UDC (Figures 4 and 5). The data performed in these Figures confirm our previous suggestions that UV irradiation may produce free radicals in bulk samples and also in pharmaceutical formulations of studied bile acids (DH and UDC, resp.). Prolonged time of exposition of DH and also UDC to UV irradiation can cause the increase of free radical concentrations in pharmaceutical formulations of both substances. Small difference between the free radical concentrations obtained for bulk samples of DH and UDC and their commercial products could be explained by

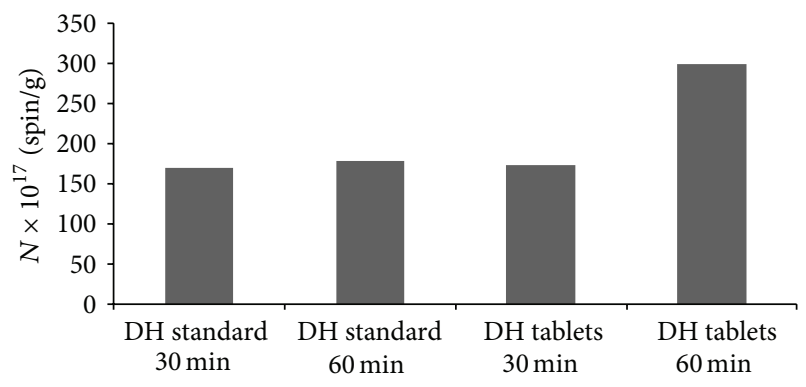

Figure 4: Comparison of free radical concentrations $(N)$ in the UV irradiated dehydrocholic acid (reference standard and tablets containing DH) during 30 and 60 minutes.

influence of other ingredients coming from both preparations on EPR measurements of investigated bile acids as active substances in analyzed drugs (tablets and capsules).

Besides described UV irradiation, the most commonly applied factor in the process of sterilization of various pharmaceutical products and bulk substances is the increased temperature. This parameter is recommended similarly like UV irradiation by Pharmacopeia (in chapter Sterility Tests) for the process of drug sterilization [14]. An achievement of thermal sterilization is relatively low cost of this method in comparison with UV irradiation. Lack of the accurate data regarding the effect of thermal sterilization on the properties and concentration of free radicals in examined dehydrocholic and ursodeoxycholic acids caused that the aim of our further studies was to determine the influence of increased temperature and also the time of thermal sterilization on $\mathrm{DH}$ 
TABLE 2: Parameters of the EPR spectra of thermally sterilized dehydrocholic acid (DH) and also ursodeoxycholic acid (UDC).

\begin{tabular}{|c|c|c|c|c|c|c|c|c|}
\hline Compound & Conditions & $\begin{array}{c}A \text { [a. u.] } \\
{[ \pm 0.02]}\end{array}$ & $\begin{array}{l}I \text { [a. u.] } \\
{[ \pm 0.02]}\end{array}$ & $\begin{array}{c}\Delta B_{\mathrm{pp}} \\
{[\mathrm{mT}]} \\
{[ \pm 0.02]}\end{array}$ & $\begin{array}{c}N \times 10^{16} \\
{[\mathrm{spin} / \mathrm{g}]} \\
{[ \pm 0.02]}\end{array}$ & $\begin{array}{l}A_{1} / A_{2} \\
{[ \pm 0.02]}\end{array}$ & $\begin{array}{c}B_{1} / B_{2} \\
{[ \pm 0.02]}\end{array}$ & $\begin{array}{c}g \\
{[ \pm 0.0002]}\end{array}$ \\
\hline \multirow{3}{*}{$\mathrm{DH}$} & $\begin{array}{c}160^{\circ} \mathrm{C} \\
(120 \text { minutes })\end{array}$ & 0.04 & 0.04 & 0.15 & 1.20 & 0.95 & 0.47 & 1.9983 \\
\hline & $\begin{array}{c}170^{\circ} \mathrm{C} \\
(60 \text { minutes })\end{array}$ & 0.02 & 0.01 & 0.13 & 0.50 & 1.00 & 0.34 & 1.9979 \\
\hline & $\begin{array}{c}180^{\circ} \mathrm{C} \\
\text { (30 minutes) }\end{array}$ & 0.07 & 0.09 & 0.10 & 2.80 & 1.34 & 0.65 & 1.9954 \\
\hline \multirow{3}{*}{ UDC } & $\begin{array}{c}160^{\circ} \mathrm{C} \\
\text { (120 minutes) }\end{array}$ & 0.08 & 0.50 & 0.42 & 15.30 & 0.61 & 1.56 & 1.9985 \\
\hline & $\begin{array}{c}170^{\circ} \mathrm{C} \\
(60 \text { minutes })\end{array}$ & 0.36 & 2.52 & 0.44 & 58.20 & 0.88 & 0.92 & 1.9982 \\
\hline & $\begin{array}{c}180^{\circ} \mathrm{C} \\
(30 \text { minutes })\end{array}$ & 0.45 & 5.21 & 0.55 & 122.30 & 0.90 & 0.98 & 1.9961 \\
\hline
\end{tabular}

Notes. $A$-amplitude of signal; $I$-integral intensity; $A_{1} / A_{2}$ and $B_{1} / B_{2}$-the asymmetry parameters; $\Delta B_{\mathrm{pp}}$-line-width of EPR signal; $N-$ free radical concentrations; $g$-factor.

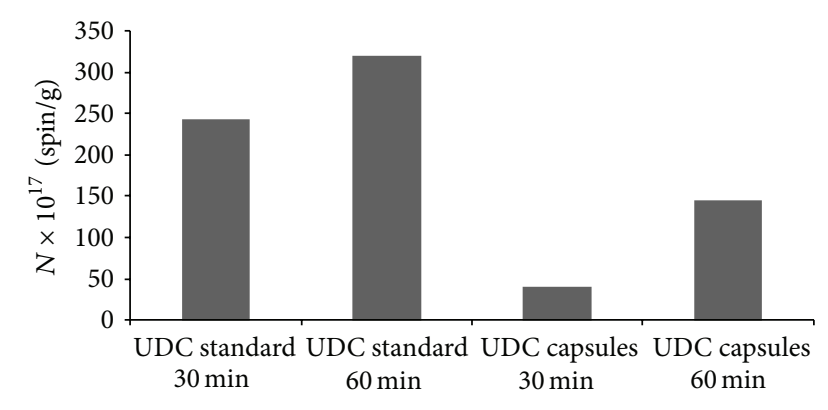

FIGURE 5: Comparison of free radical concentrations $(N)$ in the UV irradiated ursodeoxycholic acid (reference standard and tablets containing UDC) during 30 and 60 minutes.

and UDC spectroscopic properties. Analogously like in the case of the first stage of this experiment, the EPR measurements were carried out at room temperature for thermally sterilized and nonthermally sterilized samples of dehydrocholic and also ursodeoxycholic acids in solid state. Sterilization was done in accordance with Sterility Test (taken from Pharmacopoeia) at the following temperatures: $160^{\circ} \mathrm{C}, 170^{\circ} \mathrm{C}$, and $180^{\circ} \mathrm{C}$ during 120 minutes, 60 minutes, and 30 minutes, respectively [14]. EPR lines were not observed for nonthermally sterilized DH and UDC. Stable free radicals did not exist in both examined bile acids which were not operated at high temperature. Therefore, EPR spectra were recorded for thermally sterilized samples only. The exemplary EPR spectra of thermally sterilized DH and UDC are performed in Figure 6. Amplitude, integral intensity, linewidth, asymmetry parameters: $A_{1} / A_{2}, B_{1} / B_{2}$, and also the concentration of free radicals obtained under different conditions (at various temperature and during different time of sterilization) were determined and summarized in Table 2. Analysis of EPR lines indicates that the process of thermal sterilization has impact on the generation of complex free radicals systems in tested compounds. EPR spectra obtained in this experiment are asymmetric. The width of EPR lines less than $1 \mathrm{mT}$ confirmed weak dipole-dipole interactions in the studied bile acids in relation to those which have been obtained during UV irradiation sterilization. Amplitudes $(A)$ which are shown in Table 2 increase significantly with the time of sterilization in the case of ursodeoxycholic acid. Relatively less changes of this parameter are observed for dehydrocholic acid. Depending on the applied temperature, the concentration of free radicals in investigated dehydrocholic acid is placed in the range of $0.50 \times 10^{16} \mathrm{spin} / \mathrm{g}\left(\right.$ at $170^{\circ} \mathrm{C}$ ) to $2.80 \times 10^{16} \mathrm{spin} / \mathrm{g}$ (at $180^{\circ} \mathrm{C}$ ) (Table 2). The free radicals formed in sterilized ursodeoxycholic acid at the same conditions like for $\mathrm{DH}$, ranges from $15.3 \times 10^{16} \mathrm{spin} / \mathrm{g}\left(\right.$ at $160^{\circ} \mathrm{C}$ ) to $122.3 \times 10^{16} \mathrm{spin} / \mathrm{g}$ $\left(\right.$ at $\left.180^{\circ} \mathrm{C}\right)$. It could be noted that the increased temperature caused the observed growth of free radical concentrations in the examined compounds. Significant increase of $N$ value is observed for the samples which were sterilized at the highest temperature of all applied in this study (e.g., at $180^{\circ} \mathrm{C}$ ). From this fact arises the conclusion that the optimal temperature for thermal sterilization of these steroids should be $170^{\circ} \mathrm{C}(60$ minutes) in the case of $\mathrm{DH}$ and also $160^{\circ} \mathrm{C}(120$ minutes) for UDC. Under these conditions the formation of less amount of free radicals in the sterilized bile acid samples is observed.

Summing up, it could be noted that the kinds of sterilization (e.g., thermal or UV irradiation) which are widely applied in pharmacy in order to achieve appropriate microbiological purity of pharmaceutical formulations containing bile acids, such as DH and UDC, may influence the properties and also concentration of free radicals generated in these compounds during respective sterilization. As one can see on the basis of $N$ values (free radical concentration) obtained for examined compounds by two applied sterilizations, thermal sterilization is better because it allows generating less amount of free radicals in both tested compounds. Thus, this method should be applied in practice. Moreover, this technique is easy to use and cheap in comparison with UV irradiation sterilization. Therefore, UV irradiation sterilization is proposed as the second (alternative) method for bile acid samples. 


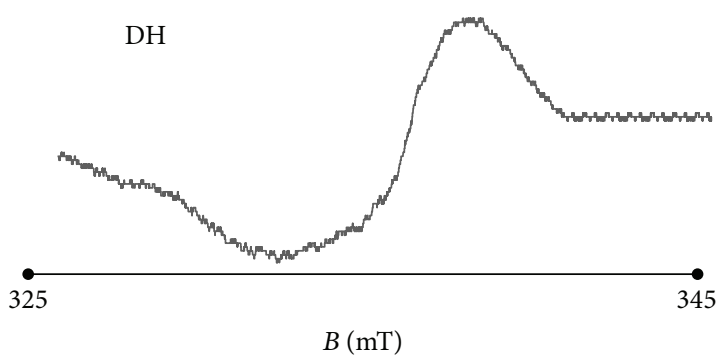

(a)

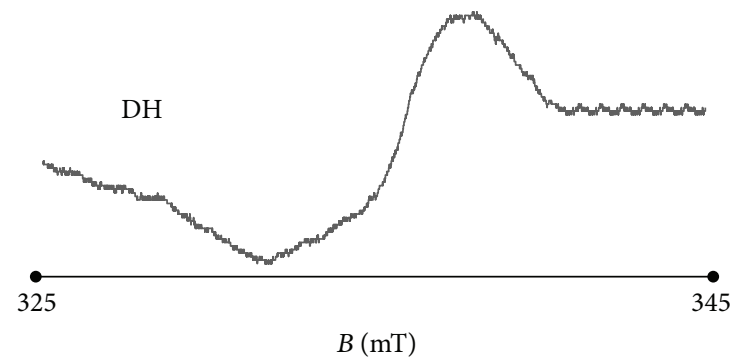

(b)

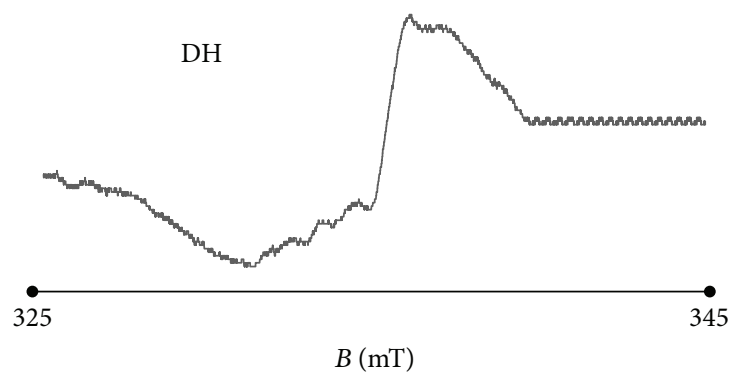

(c)

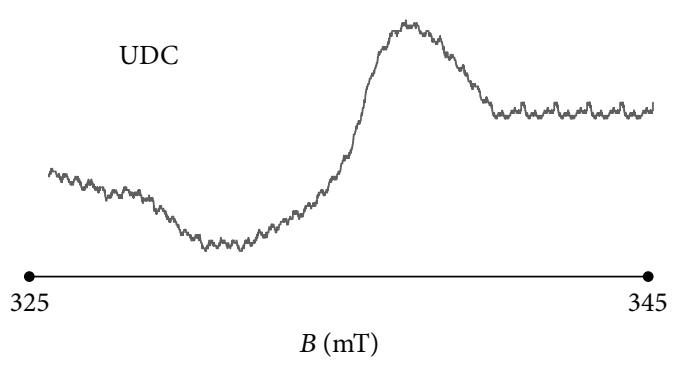

(d)

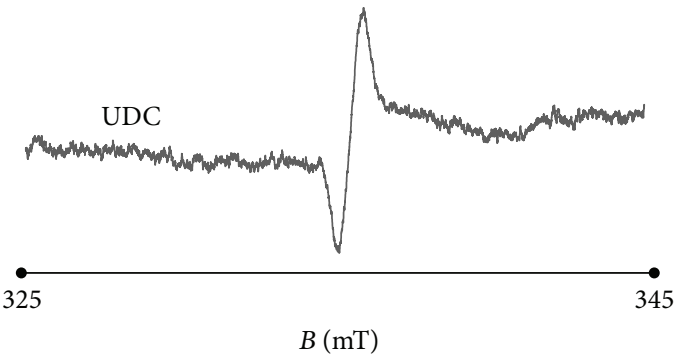

(e)

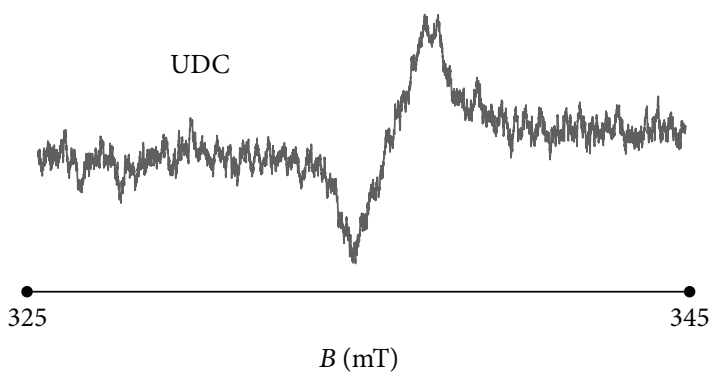

(f)

FIGURE 6: EPR spectra of DH and UDC thermally sterilized at $160^{\circ} \mathrm{C}$ : (a) and (d); at $170^{\circ} \mathrm{C}$ (b) and (e); at $180^{\circ} \mathrm{C}(\mathrm{c})$ and (f).

Our researches confirmed that EPR spectroscopy is useful to detect the formation of free radicals caused by exposure of examined bile acids to UV irradiation and also to high temperature during conducted sterilization of drug samples. Additionally, the performed EPR study allowed finding the optimal conditions of UV irradiation and thermal sterilization of tested DH and UDC which should be applied in practice.

The chemical structures of examined bile acids (in Figure 1) and also the differential EPR spectra presented in Figures 3 and 6, respectively, prove that the used sterilization conditions can change the chemical structure of tested drug substances. As it is known various chemical bonds may be ruptured in chemical structure of organic compounds during heating or by UV irradiation. It resulted in formation of different free radicals in molecule. We suppose that in the case of thermally and UV irradiated bile acid samples (DH and UDC), existence of carbon radicals could be observed, but the way of their formation may be different in both cases. For the first applied technique (UV irradiation sterilization) we can suggest degradation of steroid nucleus (saturated tetracyclic hydrocarbon perhydrocyclopentanophenanthrene) which exists in chemical structure of tested compounds.
The main source of measured carbon radicals may be the methyl groups presented in perhydrocyclopentanophenanthrene. Similar mechanism for the formation of carbon radicals in both bile acids (connected probably with the degradation of their perhydrocyclopentanophenanthrene ring) could be expected in the case of thermal sterilization of examined steroid compounds. These conclusions confirm the presence of the characteristic stretching vibrations placed in the range of $2948 \div 2875 \mathrm{~cm}^{-1}$ observed on IR spectra of sterilized bile acids obtained in our preliminary IR study of nonsterilized and sterilized samples [18]. The presented results will be an inspiration for further spectroscopic study of sterilized bile acids. They will be continued in terms of determining the accurate mechanism of formation of the carbon radicals produced during thermal and UV irradiation sterilization of tested bile acids. In addition to described IR method, other spectroscopic techniques including modern EIMS and MS/MS will be applied.

\section{Conclusions}

The performed EPR spectroscopic studies of dehydrocholic and ursodeoxycholic acids indicated the following. 
(i) UV irradiation and also increased temperature can influence on the formation and also properties of free radicals in sterilized drug samples.

(ii) The EPR measurements prove the existence of complex free radicals systems in sterilized dehydrocholic and ursodeoxycholic acids.

(iii) Free radical concentrations in examined bile acids depend on sterilization conditions, such as the temperature and the time of sterilization.

(iv) EPR spectra are useful to examine the optimal conditions of thermal and UV irradiation sterilizations of tested compounds.

(v) Taking into account the lowest free radical concentrations (the optimal thermal sterilization) is the process at temperature $160^{\circ} \mathrm{C}$ (120 minutes) for UDC and $170^{\circ} \mathrm{C}$ (60 minutes) for $\mathrm{DH}$.

(vi) The optimal time of UV irradiation sterilization for both bile acids should be 30 minutes.

(vii) EPR spectroscopic study demonstrates that, of two applied methods, thermal sterilization of examined bile acids produces less amount of free radicals (probably carbon radicals) in comparison with UV irradiation technique. Therefore, this method should be used in practice.

\section{Disclosure}

The authors submitted this paper in a much elaborate manner by mentioning the trademarks or company names, in order to make each and every point or sentence clear or transparent. The usage of this trade mark symbol or company name is for proving the genuineness of the work and not for any another purpose.

\section{Conflict of Interests}

The authors declare that there is no conflict of interests regarding the publication of this paper. They have no conflict of interests or no financial gains in mentioning the company names or trademarks. As the authors of the paper, they do not have any financial relation with the commercial identity mentioned in our paper.

\section{Acknowledgment}

This research was financed by the Medical University of Silesia as part of statutory research project in 2014 year, Project no. KNW-1-006/N/4/0 directed by Professor Alina Pyka.

\section{References}

[1] J. E. Wertz and J. R. Bolton, Electron Spin Resonance: Elementary Theory and Practical Applications, Chapman \& Hall, New York, NY, USA, 1986.

[2] G. R. Eaton, S. S. Eaton, and K. M. Salikhov, Foundations of Modern EPR, World Scientific, Singapore, 1998.
[3] J. A. Weil and J. R. Bolton, Electron Paramagnetic Resonance: Elementary Theory and Practical Applications, John Wiley \& Sons, New Jersey, NJ, USA, 2007.

[4] J. Stankowski and W. Hilczer, Introduction to Magnetic Resonance Spectroscopy, Scientific Publishing, Warsaw, Poland, 2005.

[5] K. Zawada, "EPR spectroscopy in pharmacy and medicine," Polish Pharmacy, vol. 65, no. 3, pp. 224-228, 2009.

[6] A. Skowronska, M. Wojciechowski, P. Ramos, B. Pilawa, and D. Kruk, "ESR studies of paramagnetic centers in pharmaceutical materials-cefaclor and Clarithromycin as an example," Acta Physica Polonica A, vol. 121, no. 2, pp. 514-517, 2012.

[7] M. Kościelniak-Ziemniak and B. Pilawa, "Application of EPR spectroscopy for examination of free radical formation in thermally sterilized betamethasone, clobetasol, and dexamethasone," Applied Magnetic Resonance, vol. 42, no. 4, pp. 519-530, 2012.

[8] P. Ramos, B. Pilawa, and E. Stroka, "EPR studies of free radicals in thermally sterilized famotidine," Nukleonika, vol. 58, no. 3 , pp. 413-418, 2013.

[9] B. Marciniec, K. Dettlaff, W. Danikiewicz, G. Spólnik, E. Jaroszkiewicz, and M. Naskrent, "Radiostability of ketoconazole in the solid state," Current Pharmaceutical Analysis, vol. 9, no. 1, pp. 102-113, 2013.

[10] P. Olczyk, P. Ramos, M. Bernaś, K. Komosinska-Vassev, J. Stojko, and B. Pilawa, "Application of electron paramagnetic resonance spectroscopy to comparative examination of different groups of free radicals in thermal injuries treated with propolis and silver sulphadiazine," Evidence-based Complementary and Alternative Medicine, vol. 2013, Article ID 851940, 11 pages, 2013.

[11] E. Buszman, B. Betlej, D. Wrześniok, and B. RadwańskaWala, "Effect of metal ions on melanin-local anaesthetic drug complexes," Bioinorganic Chemistry and Applications, vol. 1, no. 2, pp. 113-122, 2003.

[12] P. Olczyk, P. Ramos, M. Bernas, K. Komosinska-Vassev, J. Stojko, and B. Pilawa, "Microwave saturation of complex EPR spectra and free radicals of burnt skin treated with apitherapeutic agent," Evidence-Based Complementary and Alternative Medicine, vol. 2013, Article ID 545201, 9 pages, 2013.

[13] A. Kaczmarek, J. Cielecka-Piontek, P. Garbacki et al., "Radiation sterilization of anthracycline antibiotics in solid state," The Scientific World Journal, vol. 2013, Article ID 258758, 7 pages, 2013.

[14] Polish Pharmacopoeia, Polish Pharmaceutical Society, Warsaw, Poland, 2013.

[15] K. R. Sharma, "Review on bile acid analysis," International Journal of Pharmaceutical and Biomedical Science, vol. 3, no. 2, pp. 28-34, 2012.

[16] S. Mukhopadhyay and U. Maitra, "Chemistry and biology of bile acids," Current Science, vol. 87, no. 12, pp. 1666-1683, 2004.

[17] J. Sjövall, W. J. Griffiths, K. D. R. Setchell, N. Mano, and J. Gotoin, Analysis of Bile Acids in Steroid Analysis, Springer, London, UK, 2nd edition, 2010.

[18] R. M. Silverstein, F. X. Webster, and D. J. Kiemle, Spectrometric Identification of Organic Compounds, John Wiley \& Sons, New York, NY, USA, 7th edition, 2005. 

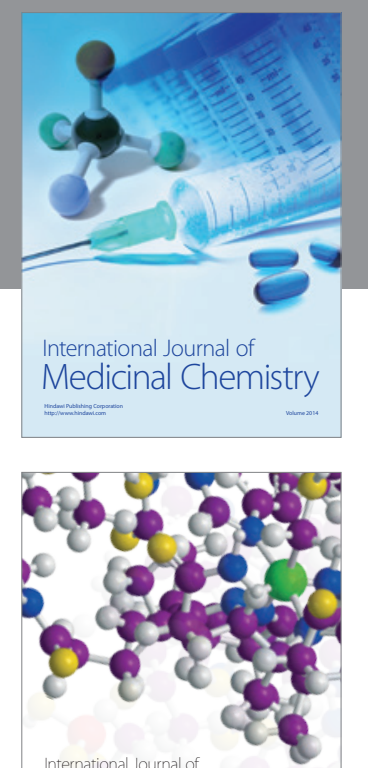

\section{Carbohydrate} Chemistry

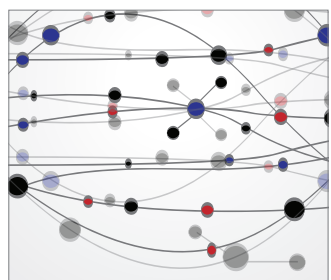

The Scientific World Journal
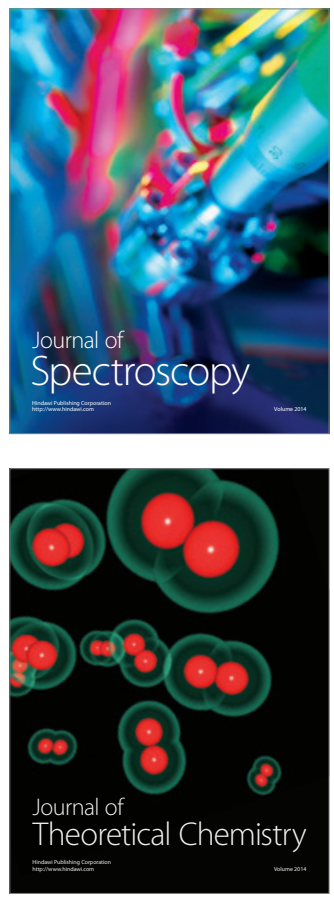
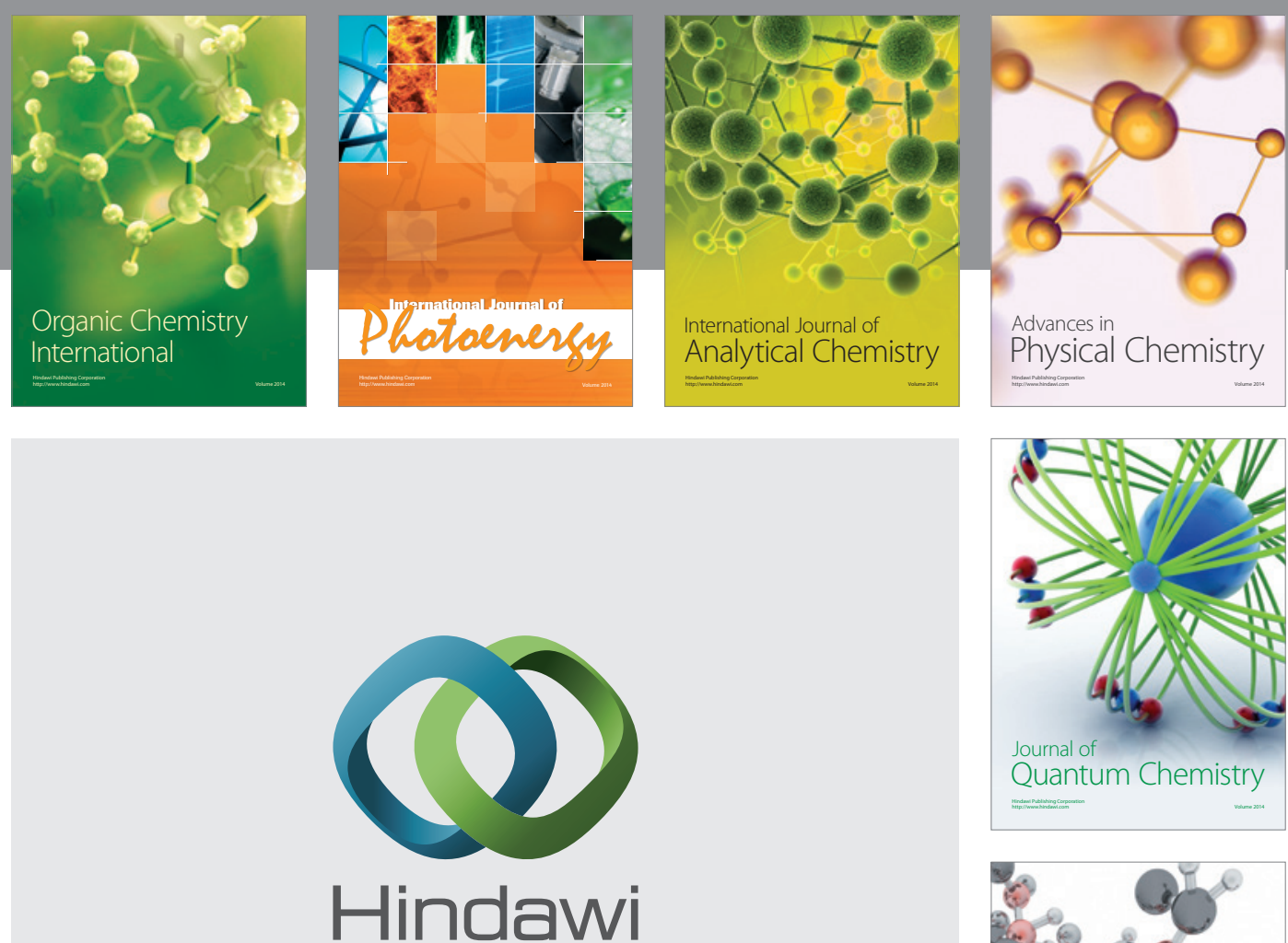

Submit your manuscripts at

http://www.hindawi.com

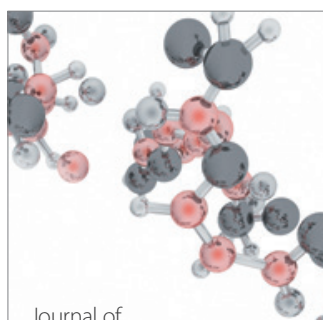

Analytical Methods

in Chemistry

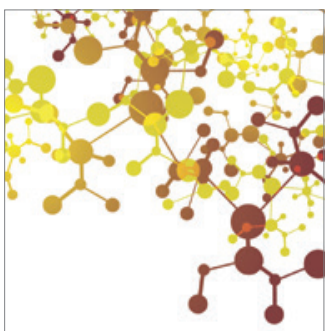

Journal of

Applied Chemistry

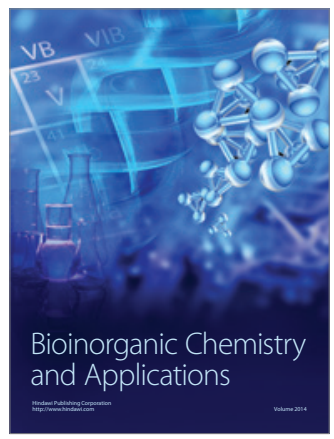

Inorganic Chemistry
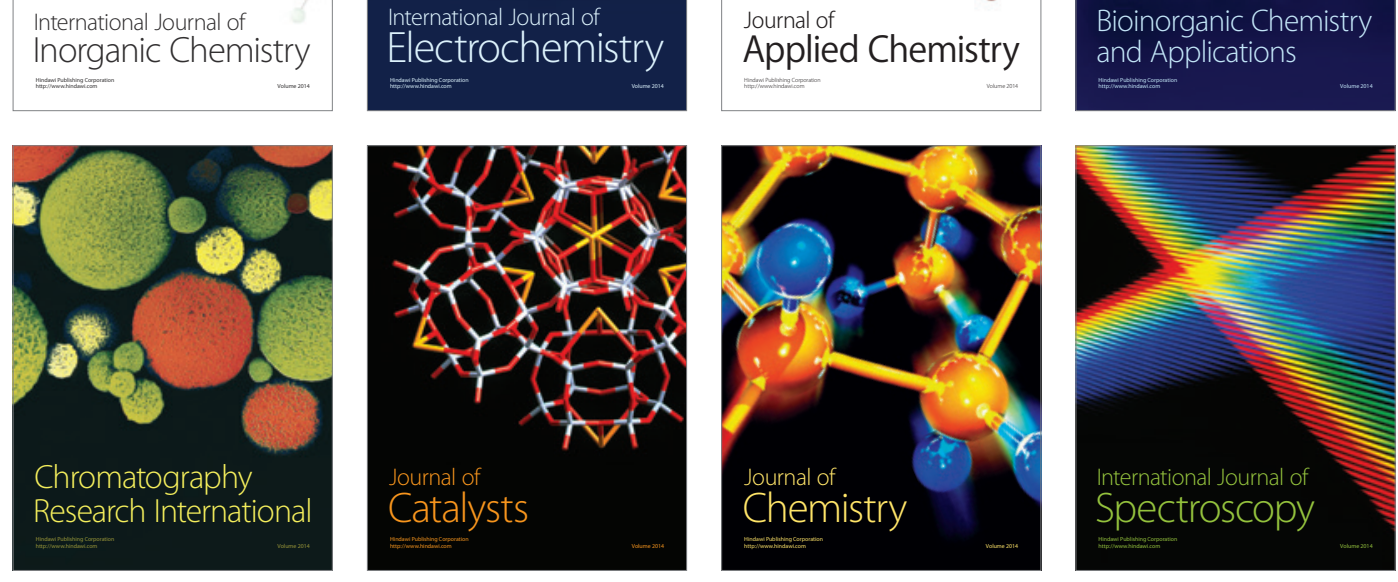